\title{
ON THE CONVERGENCE OF SERIES
} WITH RECURSIVELY DEFINED TERMS

\begin{abstract}
N. S. HOANG
Abstract. We investigate the asymptotic behavior of a sequence $\left(x_{n}\right)_{n=0}^{\infty}$ defined recursively by $x_{n+1}=f\left(x_{n}\right), n \geqslant 0$ where $f:[0, \infty) \rightarrow[0, \infty)$ is a continuous function. A fundamental criterion on the function $f(x)$ for estimating the rate of decay of $x_{n}$ as $n$ tends to $\infty$ and for testing convergence of the series $\sum_{n=0}^{\infty} x_{n}$ is proposed and justified. Criteria for testing absolute and conditional convergence of $\sum_{n=0}^{\infty} x_{n}$ when $f(x)$ is not a non-negative function are also formulated and proved.
\end{abstract}

Mathematics subject classification (2010): 40A05, 40F05.

Keywords and phrases: Convergence, series, rate of decay, comparison test.

\section{REFERENCES}

[1] T. Andreescu and R. Gelca, Putnam and Beyond, Springer-Verlag, New York, 2007.

[2] E. R. Easdale, J. E. Fleming, And B. D. Suceava, Convergence for Series With Terms Defined by a Recurrence Relation, Amer. Math. Monthly 124 (2017) 360-364.

[3] J. E. Harris And B. D. Suceava, Problem 11244, Amer. Math. Monthly 113 (2006) 759-760. 